\title{
A gut instinct
}

The gastrointestinal tract is the major site of nutrient absorption in the body and plays a central role in the pathogenesis of many systemic diseases, including type 2 diabetes. The small intestine in particular has been recognized as an important therapeutic target to regulate sugar uptake into the blood. Now, writing in Nature Materials, Jeffrey Karp, Yuhan Lee, Ali Tavakkoli and colleagues report a biomaterial that can be ingested as a pill and that forms a transient, yet continuous coating on a portion of the intestine, modulating nutrient exposure and thus the transport of glucose into the blood, and provides a protective vehicle for drugs.

Type 2 diabetes is a leading cause of blindness, amputations, renal failure and poor cardiovascular outcomes, and is characterized by high levels of blood glucose,

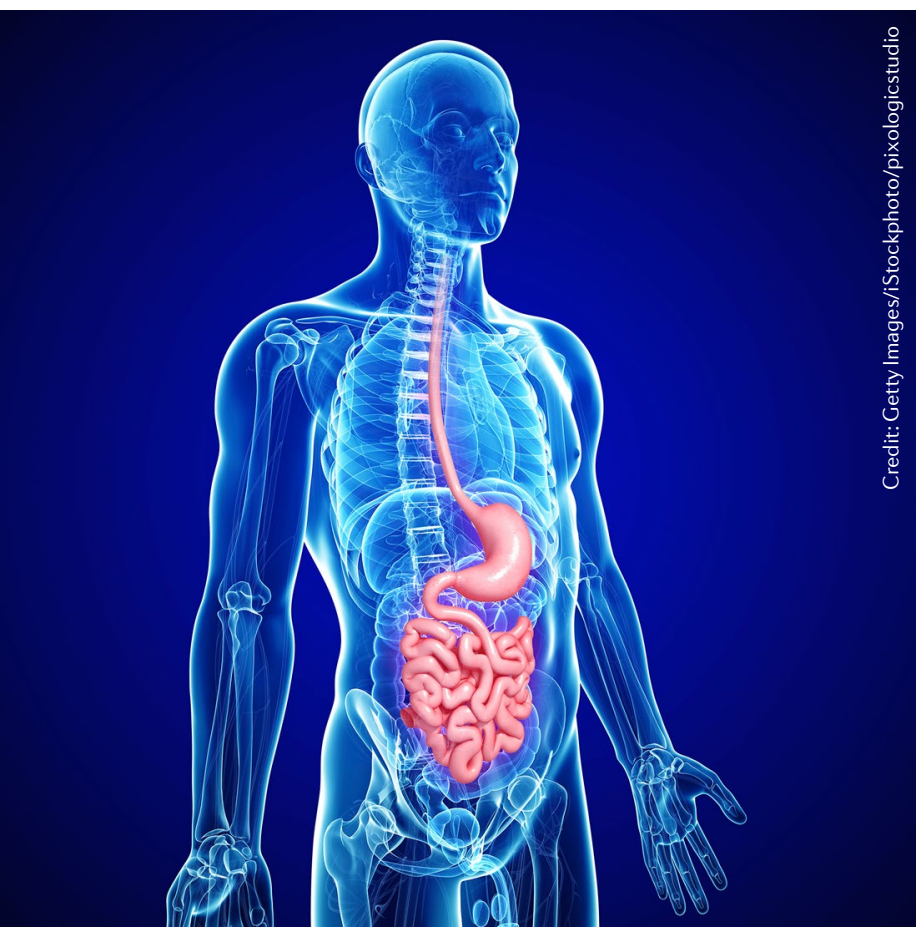

which must cross the barrier of the small intestine to enter the bloodstream. Thus, modulating nutrient exposure to the small intestine would offer a therapeutic strategy for the treatment of type 2 diabetes. "Bariatric surgery, bypassing the stomach and part of the intestine from the food stream, has shown very promising results, but the high body mass index requirements to qualify and risks of surgery along with permanent changes to gastrointestinal anatomy deter many patients from surgery," explains Karp. "Therefore, there is an urgent need for a safe, non-invasive and effective treatment for a wider diabetic patient population." However, directing therapeutic agents to the small intestine through oral drug delivery is challenging and usually requires systemic administration. Moreover, the highly acidic and proteolytic environment of the stomach can affect drug activity.

The US Food and Drug Administration (FDA)-approved drug, sucralfate, contains anioinic sucrose octasulfate and a cationic polyaluminium complex, which, when exposed to acidic $\mathrm{pH}$ in the stomach, form a sticky paste. Karp and colleagues engineered this material into a coacervate assembled by $\mathrm{pH}$-independent electrostatic interactions, making it reversibly (de)hydratable and attach to both healthy and diseased intestinal mucosa. Therefore, the material can be ingested as an oral drug in the form of a powder and, once hydrated, establishes a continuous coating along the inner wall of the intestine, providing a barrier to the uptake of nutrients. By adjusting the viscosity of the material, the location, extent and durability of the coating can be tailored. "We envisioned a pill that a patient can take before a meal that transiently coats the gut to replicate the effects of bariatric surgery," says Tavakkoli.

Karp and colleagues have tested the material - termed LuCI (Luminal Coating of the Intestine) - in vitro using a model mucous membrane under dynamic conditions, demonstrating that $\sim 80 \%$ of glucose transport can be blocked by the material. The researchers showed in rats that the coating remained effective for 2-3 hours after oral delivery. Moreover, upon intake of glucose, the material acts as a nutrient barrier and significantly reduces the blood glucose response in the rats. "LuCI is a derivative of a very safe FDA approved drug used in millions of patients and exhibits an excellent safety profile without systemic absorption," comments Karp. "We have shown a robust response in modulating glucose absorption in rats and our next goal is to test $\mathrm{LuCI}$ in a diabetic model and in humans to demonstrate long-term impact on the remission of type 2 diabetes."

The team also tested $\mathrm{LuCI}$ for its potential to selectively deliver drugs to the small intestine. The powder formulation of the material allows the mixing of drugs, including proteins, with $\mathrm{LuCI}$ and subsequent transport to the small intestine. Delivery with LuCI maintains the activity of the drug in the stomach and enables its gradual local release throughout the entire gastrointestinal tract, making it a promising candidate as a drug delivery vehicle.

Christine-Maria Horejs

ORIGINAL ARTICLE Lee, Y. et al. Therapeutic luminal coating of the intestine. Nat. Mater. https://doi.org/10.1038/s41563-018-0106-5 (2018) 